\title{
INVARIANT TRACES ON ALGEBRAS
}

\author{
GUIDO KARRER
}

\begin{abstract}
Certain properties of traces on a finite-dimensional associative algebra $A$ lead to the definition of an element $t(A) \in$ $H^{1}$ (Out $\left.A, C^{*}\right), C^{*}$ being the multiplicative group of the center of $A$ as Out $A$-module. It is shown that $t(A)=0$ is equivalent to the existence of nondegenerate traces on $A$ which are invariant under composition with all automorphisms of $A$. In particular, by means of Galois theory, $t(A)=0$ is shown for a semisimple algebra $A$, whereas $t(A) \neq 0$ for certain group algebras.
\end{abstract}

1. Let $R$ be a field, $A$ an associative unitary algebra of finite dimension over $R$. By a trace on $A$ we mean a linear map $\tau: A \rightarrow R$ such that $\tau(a b)=\tau(b a) \forall a, b \in A$. This is one possible generalization of the notion of a trace on matrix rings (see [4]; for a generalization in another context, see [2]).

In $\S \S 2-4$ we shall list some generalities on traces; let $T(A)$ be the $R$-vectorspace of all traces on $A$.

2. The existence of nonzero traces on $A$ depends on the abelianized algebra $A^{a}$. Let $[A, A]$ be the vectorspace generated by all commutators $[a, b]=a b-b a$ in $A, A^{a}$ the quotient $A /[A, A]$. The class map $\pi: A \rightarrow A^{a}$ provides an isomorphism of vectorspaces

$$
\pi^{*}: \operatorname{Hom}_{R}\left(A^{a}, R\right) \rightarrow T(A),
$$

where $\pi^{*}$ is the dual map of $\pi$.

One knows that $A^{a} \neq(0)$ if $A$ is simple [1], hence

$$
T(A) \neq(0) \text { for a simple algebra } A \text {. }
$$

3. The radical of a trace $\tau$ is the set

$$
R_{\tau}=\{a \in A / \tau(a b)=0 \forall b \in A\},
$$

Received by the editors February 12, 1973 and, in revised form, May 17, 1973.

AMS (MOS) subject classifications (1970). Primary 16A49, 16A40; Secondary 16A26, 18A10.

Key words and phrases. Trace on algebras, semisimple algebra, group algebra, cohomology of groups, automorphism of a group algebra.

(C) American Mathematical Society 1974 
and $\tau$ is nondegenerate if $R_{\tau}=(0)$. As $R_{\tau}$ is a 2-sided ideal,

$$
\text { a nonzero trace on a simple algebra is nondegenerate. }
$$

$T(A)$ is a module over the center $C$ of $A$, as $z \cdot \tau$ for $z \in C$ and $\tau \in T(A)$ defined by

$$
(z \cdot \tau)(a):=\tau(z a), \quad a \in A,
$$

is again a trace.

Proposition 1. A nondegenerate trace $\eta \in T(A)$ is a free generator of the $C$-module $T(A)$.

Proof. $\eta$ provides a linear isomorphism from $A$ to its dual $\operatorname{Hom}_{R}(A, R)$; for every $\tau \in T(A) \subset \operatorname{Hom}_{R}(A, R)$ there exists a unique $b \in A$ such that $\tau(a)=\eta(b a) \forall a \in A$. We then have the following sequence of implications

$$
\begin{aligned}
\tau\left(a_{1} a_{2}\right) & =\tau\left(a_{2} a_{1}\right) \quad \forall a_{i} \in A \\
& \Rightarrow \eta\left(b a_{1} a_{2}\right)=\eta\left(b a_{2} a_{1}\right)=\eta\left(a_{1} b a_{2}\right) \quad \forall a_{i} \in A \\
& \Rightarrow b a_{1}-a_{1} b \in R \eta=(0) \quad \forall a_{1} \in A \\
& \Rightarrow b \in C \Rightarrow \tau=b \cdot \eta .
\end{aligned}
$$

Corollary 1. Suppose the set $B(A)$ of nondegenerate traces on $A$ is nonempty. Then, the $C$-module structure of $T(A)$ defines a simply transitive action of $C^{*}$ on $B(A)$, where $C^{*}$ is the multiplicative group of invertible elements of the center $C$ of $A$.

4. Let Aut $A$, In $A$ denote the group of all automorphisms and antiautomorphisms, of all inner automorphisms resp. of $A$, and denote the quotient group Aut $A / \operatorname{In} A$ by Out $A$. As can be seen immediately from the definitions, composing an (anti-) automorphism with a trace yields again a trace and thus an operation of Aut $A$ on $T(A)$. Inner automorphisms act in this way as the identity, and we finally get an action of Out $A$ on $T(A)$. Let $\tau \cdot \omega(\tau \in T(A), \omega \in$ Out $A)$ be the symbol for this action. Its relationship with the $C$-module structure of $T(A)$ may be described in the form of an associative law

$$
(\omega c) \cdot\left(\tau \cdot \omega^{-1}\right)=(c \cdot \tau) \cdot \omega^{-1}, \quad c \in C, \tau \in T(A), \omega \in \text { Out } A .
$$

5. We say that a trace $\tau$ is invariant if $\tau \cdot \omega=\tau \forall \omega \in$ Out $A$. We are coming now to the main point of this note which consists in giving a condition on the cohomology level for the existence of nondegenerate invariant traces.

In the subsequent statement, $C^{*}$ is meant to be an Out $A$-module via the operation of automorphisms on the center. 
Proposition 2. For every algebra $A$ with $B(A) \neq \varnothing$, there is defined an element $t(A) \in H^{1}\left(\right.$ Out $\left.A, C^{*}\right)$ such that $t(A)=0$ precisely if $A$ has nondegenerate invariant traces.

Proof. By Corollary 1, there belongs to every $\tau \in B(A)$ a map $f_{r}:$ Out $A \rightarrow C^{*}$ such that

$$
\tau \cdot \omega^{-1}=f_{\tau}(\omega) \cdot \tau \quad \forall \omega \in \text { Out } A .
$$

Then, the following statements are immediate consequences of (4.1):

(1) $f_{\tau}$ is a crossed homomorphism.

(2) For $\tau$ and $\eta \in B(A), f_{\tau}$ and $f_{\eta}$ differ by a principal crossed homomorphism.

If $t(A)$ is then defined as the cohomology class of the $f_{\tau}$ 's the statement in Proposition 2 on $t(A)$ is easily verified using again (4.1).

6. Example 1. $t(A)=0$ for a semisimple algebra $A$. In fact, if $A$ is simple we know from (2.1) and (3.1) that $B(A) \neq \varnothing$. As Out $A$ is a finite group and $C^{*}$ the multiplicative group of a field, a fundamental theorem of Galois theory asserts that $H^{1}\left(\mathrm{Out} A, C^{*}\right)=0$ [3, Chapter IV, p. 106]. By Proposition 2, $A$ has nondegenerate invariant traces.

If $A=\bigoplus A_{i}(1 \leqq i \leqq n)$ is semisimple with simple components $A_{i}$, let

$$
\text { Out }{ }_{i} A:=\left\{\omega \in \text { Out } A / \omega\left(A_{i}\right) \subset A_{i}\right\} \text {. }
$$

Choose one index $i$ for each conjugacy class of the subgroups Out ${ }_{i} A \subset$ Out $A$, and on $A_{i}$ a nondegenerate invariant trace $\tau_{i}$. If Out ${ }_{k} A$ is conjugate to Out ${ }_{i} A$, there exists $\alpha \in$ Aut $A$ with $\alpha: A_{k} \rightarrow A_{i}$, and define $\tau_{k}$ on $A_{k}$ by $\tau_{k}=\tau_{i} \circ \alpha$. The direct sum of all these traces on the different $A_{i}$ is seen to be a nondegenerate invariant trace on $A$.

7. Example 2. Let $G_{p}$ be a finite cyclic group of prime order $p>2$, $R=Z_{p}$ and $A$ the group algebra $Z_{\nu}\left(G_{p}\right)$. Then, $t(A) \neq 0$.

First we note, that in the more general situation of a finite group $G$ and field $R$, the group algebra $R(G)$ has at least one nondegenerate trace $\tau_{0}$ given by $\tau_{0}(x)=x(1)$ where $x=\sum x(g) \cdot g \in R(G), g \in G$ and $x(g) \in R$, and 1 is the unit in $G$. Hence, $t(R(G))$ is defined.

Suppose now $q$ is a generator of $G_{p}$. As $A=Z_{p}\left(G_{p}\right)$ is commutative, we have Out $A=$ Aut $A$ and every $\alpha \in$ Aut $A$ is characterized by its value on $q$. If $x=\alpha(q), x^{p}=1$ and the powers $x^{v}, 0 \leqq v \leqq p-1$, form an $R$-basis of $A$. Conversely, every $x \in A$ with this property is the value of some $\alpha \in$ Aut $A$ on $q$. Therefore at least $p$ automorphisms $\alpha_{v}, 0 \leqq v \leqq p-1$, of $A$ exist which are given by their values on $q$ :

$$
\begin{aligned}
& \alpha_{v}(q)=q^{v}, \quad 1 \leqq v \leqq p-1, \\
& \alpha_{0}(q)=\frac{1}{2}(1+q) .
\end{aligned}
$$


From this we conclude that an invariant trace $\tau$ on $A=Z_{p}\left(G_{p}\right)$ must assume the same value on each $q^{v}, 0 \leqq v \leqq p-1$, and as such must be a multiple of the augmentation $\varepsilon: Z_{p}\left(G_{p}\right) \rightarrow Z_{p}$. The kernel of $\varepsilon$ being an ideal, $\varepsilon$ is a degenerate trace and so is $\tau$.

\section{REFERENCES}

1. B. Harris, Commutators in division rings, Proc. Amer. Math. Soc. 9 (1958), 628-630. MR 20\#3180.

2. A. Hattori, Rank element of a projective module, Nagoya Math. J. 25 (1965), 113-120. MR 31 \#226.

3. S. Mac Lane, Homology, Die Grundlehren der math. Wissenschaften, Band 114, Academic Press, New York; Springer-Verlag, Berlin, 1963. MR 28 \#122.

4. B. L. van der Waerden, Algebra. Teil 1, Siebte Auflage, Heidelberger Taschenbücher, Band 12, Springer-Verlag, Berlin and New York, 1966. MR 41 \#8186.

Department of Mathematics, University of Zurich, Zurich, Switzerland 